\title{
EVALUACIÓN DE LA SEGURIDAD ALIMENTARIA SOSTENIBLE EN EL MAGREB CENTRAL CON INDICADORES AGREGADOS
}

\author{
José Soler $^{1}$ y Juan Manuel Arroyo ${ }^{2}$ \\ Escuela de Ingeniería Agrícola, UPM
}

\begin{abstract}
Resumen:
Se ha desarrollado un indicador agregado mediante la técnica de análisis de componentes principales, que incluye 21 indicadores individuales para 60 países. Los países del Magreb Central no muestran una seguridad alimentaria sostenible de acuerdo a las variables estudiadas y dentro del conjunto de países incluidos en este trabajo. Aunque la producción de alimentos es relativamente sostenible desde el punto de vista ambiental, no lo es en las dimensiones humana y productiva, ya que los cereales se presentan como el mayor aporte energético en la dieta, si bien, por un lado, no cubren los requerimientos óptimos y, por otro, la productividad de su cultivo en el Magreb Central es deficiente, debiéndose cubrir con importaciones externas.
\end{abstract}

Palabras clave: Indicadores agregados, Magreb central, seguridad alimentaria.

Title in English: “Assessing Sustainable Food Security in the Central Maghreb through Aggregated Indicators"

\begin{abstract}
:
An aggregated indicator has been developed using the multivariate statistical tool of principal components analysis, with a matrix of 60 countries x 21 variables. Central Maghreb countries don't show sustainable food security within the countries and variables included in this study. Although food production shows a relatively environmental sustainability, the social and productive dimensions are relatively unsustainable because cereals represent a great amount of energy in diets but optimum dietary requirements and not properly fulfilled and cereal production and yields in these countries are low and quite variable, so they depend on external imports.
\end{abstract}

Keywords: Aggregated Indicators, Central Maghreb, Food Security.

Copyright (C) UNISCI, 2013.

Las opiniones expresadas en estos artículos son propias de sus autores, y no reflejan necesariamente la opinión de UNISCI. The views expressed in these articles are those of the authors, and do not necessarily reflect the views of UNISCI.

\footnotetext{
${ }^{1}$ José Soler es profesor titular del Departamento de Producción Vegetal: Fitotecnia de la Escuela de Ingeniería Técnica Agrícola de la Universidad Politécnica de Madrid y miembro del Foro Hispano-Argelino.

Dirección: Escuela de Ingeniería Técnica Agrícola UPM, Ciudad Universitaria, s/n 28040 Madrid, España E-mail: jose.soler@upm.es.

2 Juan Manuel Arroyo es profesor titular del Departamento de Producción Vegetal: Fitotecnia de la Escuela de Ingeniería Técnica Agrícola de la Universidad Politécnica de Madrid y miembro del Foro Hispano-Argelino. Dirección: Escuela de Ingeniería Técnica Agrícola UPM, Ciudad Universitaria, s/n 28040 Madrid, España E-mail: juanmanuel.arroyo@upm.es http://dx.doi.org/10.5209/rev_UNIS.2013.n31.4470
} 


\section{Introducción}

La producción sostenible de alimentos para el suministro a una población y a un consumo per cápita en crecimiento es un gran reto a medio y largo plazo ${ }^{3}$. La seguridad alimentaria incluye aspectos tales como la oferta y la demanda, así como el acceso económico y físico a los alimentos, además de la asimilación de los nutrientes y la salud ${ }^{4}$. Esta seguridad alimentaria debe alcanzarse con una producción agrícola sostenible que asegure también la productividad a largo plazo y los servicios de los ecosistemas. El Magreb Central incluye los países de Argelia, Marruecos y Túnez, situados en el Norte de África y, aparte de diferencias socioeconómicas, poseen unas características agroecológicas que limitan la productividad agrícola: climas áridos y semiáridos, con bajos niveles de precipitaciones que se concentran sobre todo en las zonas más próximas a la costa donde también están los suelos más fértiles para el cultivo.

Estos condicionantes ecológicos se suman a otros de índole política y socioeconómica que han dado lugar a una estructura agraria dual: por un lado sistemas hortofrutícolas intensivos orientados a la exportación y, por otro, sistemas cerealísticos extensivos orientados al mercado interior ${ }^{6}$. En estos sistemas la introducción de ciertos inputs, como es el caso de los fertilizantes ${ }^{7}$, es escasa, y existe una diferencia importante entre los rendimientos actuales y los potenciales. Esto hace que el suministro interno de productos básicos se supla con importaciones del exterior ${ }^{8}$, mientras que las frutas y hortalizas se exportan fuera. En este contexto, tanto España como la UE son actores clave en el comercio con el Magreb Central, y en el establecimiento de políticas agrícolas, de desarrollo, sobre alimentación, medioambientales y sobre salud.

Este reto multidimensional debe abordarse con un enfoque holístico, en el que la producción sostenible y la seguridad alimentaria en esta zona se evalúen mediante indicadores de sus fortalezas y debilidades en un escenario global y multinivel ${ }^{9}$. Estos indicadores pueden sintetizarse en un único y apropiado indicador que recoja toda la información posible y que pueda ser entendido y utilizado por sus usuarios finales (encargados de políticas, técnicos, científicos, etc.).

\footnotetext{
${ }^{3}$ Godfray, HC.; Beddington, J.R.; Crute, I.R.; Haddad, L.; Lawrence, D.; Muir, J.F.; Pretty, J.; Robinson, S.; Thomas, S.M. and Toulmin, C.:"Food Security: The Challenge of Feeding 9 Billion People", Science, $\mathrm{n}^{\circ} 327$, (Febrero 2010).

${ }^{4}$ González Torres, Francisco y Rojo Hernández, Carlos: "La Seguridad Alimentaria en Argelia: Indicadores Macroeconómicos. Producción Agrícola y Restricciones. Concepto de Seguridad Alimentaria y Vulnerabilidad a la Inseguridad Alimentaria", en Marquina, Antonio (ed.) (2012): Las Relaciones Hispano-Argelinas. Contexto Histórico, Desafios y Proyectos Comunes, Madrid, Foro Hispano-Argelino, pp. 162-172.

${ }^{5}$ Rojo Hernández, Carlos y González Torres, Francisco: "La Seguridad Alimentaria en Argelia: Distribución de Cultivos, Superficies, Rendimientos y Perspectivas de Futuro para la producción de Alimentos en Argelia", en "Las Relaciones Hispano-Argelinas...", op.cit., pp. 212-213.

${ }^{6}$ Soler Rovira, José y Arroyo Sanz, Juan Manuel: "Suministro de Alimentos, Factores Agronómicos e Indicadores Ambientales en el Magreb Central", este mismo volumen.

${ }^{7}$ Soler Rovira, José y Arroyo Sanz, Juan Manuel: "Suministro de Alimentos, Insumos, Flujo de Materiales e Indicadores Ambientales en Argelia”, en "Las Relaciones Hispano-Argelinas...", op.cit., pp. 193-198.

${ }^{8}$ Ibid., pp. 198-201.

9 Soler-Rovira, José; Arroyo-Sanz, Juan Manuel; González-Torres, Francisco; Rojo-Hernández, Carlos y Marquina Barrio, Antonio (2012): “Aggregated indicators of sustainable agricultural production and food security in the Central Maghreb", en Corson, M.S., van der Werf, H.M.G. (eds.): Book of Abstracts of the $8^{\text {th }}$ International Conference on Life Cycle Assessment in the Agri-Food Sector (LCA Food 2012), Saint-Malo, France, 2012.
} 
Los indicadores agregados ayudan a comunicar la información de modo sucinto y hacen más fácil distinguir tendencias en los datos de partida, a la vez que realizan el proceso de síntesis de esos datos mediante un procedimiento formalizado sustituyendo al proceso de agregación intuitiva y subjetiva que se suele hacer de modo implícito cuando se maneja una gran cantidad de datos ${ }^{10}$.

El proceso comienza formando un conjunto de indicadores individuales que van a formar parte del indicador agregado final. Así, se puede partir de dos concepciones de la sostenibilidad, bien sea orientada a objetivos o a propiedades. Estas propiedades sistémicas se recogen en el esquema de Bossel $^{11}$ (tabla 1) y son: existencia, efectividad, libertad de acción, seguridad, adaptabilidad, coexistencia y necesidades psicológicas. Estas propiedades forman parte de un marco jerárquico con tres subsistemas o dimensiones de la sostenibilidad: humano (dimensión social y de seguridad alimentaria), natural (dimensión ecológica o ambiental) y de soporte (dimensión económica o productiva).

De este modo, el objetivo de este trabajo es evaluar la producción sostenible de alimentos y la seguridad alimentaria en el Magreb Central mediante un indicador agregado utilizando técnicas de análisis multivariante.

\section{Metodología}

El indicador agregado se desarrolló dentro del marco sistémico de Bossel, en el que se incluyen siete orientadores básicos en cada uno de los tres subsistemas (humano, natural y productivo) resultando un total de 21 indicadores individuales. En la tabla 1 se muestran los indicadores empleados y las fuentes utilizadas.

Se incluyeron en el estudio un total de 60 países, entre los que están, además del Magreb Central (Argelia, Marruecos y Túnez), el resto del Magreb, el Máshreq, el Mediterráneo, la Unión Europea, Oriente próximo, el Sahel y los países de la OPEC (tabla 2). De este modo, se consideraron países con similitudes geográficas, agroecológicas, sociales y económicas a los del Magreb Central, así como países con cierta proximidad pero también con ciertas diferencias, como los países de la Unión Europea o los del Sahel. Esto posibilita un análisis más global y trata de incluir la diversidad mundial, permitiendo que el análisis multivariante de los datos exprese mejor las diferencias entre países y los ordene en función de su grado de seguridad alimentaria sostenible relativa.

De este modo, se construyó una matriz de datos con 21 indicadores de 60 países. A los datos de partida se asignó un signo positivo o negativo para poder hacerlos unidireccionales. Por ejemplo, la huella hídrica se signó negativamente, ya que consideramos que es positivo un menor consumo de recursos; por el contrario, el consumo de proteínas se signó positivamente

\footnotetext{
${ }^{10}$ Jollands, Nigel, Lermit, Jonathan, Patterson, Murray. “Aggregate eco-efficiency indices for New Zealand - a principal component analysis”. Journal of Environmental Management, n 73 (December 2004).

${ }^{11}$ Para una explicación más en profundidad de la base, significado y aplicación práctica de estas propiedades véanse de Hartmut Bossel las publicaciones: Bossel, Hartmut (1999): Indicators for sustainable development: Theory, method, applications, Winnipeg, International Institute for Sustainable Development. Manitoba, Canada; Bossel, Hartmut: "Policy assessment and simulation of actor orientation for sustainable development", Ecological Economics, $\mathrm{n}^{\circ} 34$ (Diciembre 2000) y Bossel, Harmut: "Assessing viability and sustainability: systems-based approach for deriving comprehensive indicator sets”, Conservation Ecology, vol. 2, n 5 (Enero 2001).
} 
ya que un mayor aporte proteico en la dieta contribuirá a una mejor seguridad alimentaria. Esta matriz de datos se sometió a un análisis estadístico de componentes principales (ACP) con el software STATGRAPHICS.

El ACP es una técnica de análisis multivariante que se usa para estudiar conjuntos de datos de cierto volumen. Reproduce la variabilidad entre las variables estudiadas usando un número menor de nuevas variables llamadas componentes principales $(\mathrm{CP})$, que son combinaciones lineales de las variables originales, en función de unos coeficientes de correlación, de modo que un mayor valor absoluto de los mismos indica que esa variable tiene un peso elevado en la creación de ese componente ${ }^{12}$. El primer componente (CP1) explica la máxima varianza entre los datos, mientras que el segundo es una nueva combinación de las variables y es ortogonal al primer $\mathrm{CP}$, explicando la segunda máxima varianza entre los datos, y así sucesivamente el tercero, cuarto, etc. La varianza explicada por cada CP se calcula mediante los eigenvalues o autovalores. Cada observación (en este caso, cada país) tendrá unas coordenadas con cada $\mathrm{CP}$, que se obtienen resolviendo la combinación lineal con sus coeficientes de correlación con los valores de cada variable concretos de ese país (con valores estandarizados de media cero y varianza uno). Estas coordenadas se pueden utilizar en el proceso de agregación del indicador y los autovalores permiten asignar un peso a cada variable, tal como se explica más adelante.

Con los resultados del ACP se calculó un indicador agregado de seguridad alimentaria sostenible del siguiente modo ${ }^{13}$ :

$$
A C P_{\text {seguridada } \lim \text { entariasostenible }}(i)=\frac{\sum_{k=1}^{j} F_{k i} \sqrt{\lambda_{k}}}{\sum_{k=1}^{j} \sqrt{\lambda_{k}}} \quad i=1, \ldots, 60(\text { países })
$$

Donde:

$\mathrm{F}_{\mathrm{ki}}$ es la coordenada del país i en el componente $\mathrm{k}$ (y se han considerado j componentes).

$\lambda_{\mathrm{k}}$ es el autovalor del componente $\mathrm{k}$.

Se calcularon también indicadores agregados para cada dimensión o subsistema estudiado (natural, humano y productivo) por separado, haciendo el análisis de componentes principales únicamente con los siete indicadores de cada subsistema, empleando el método anterior con cada coordenada y autovalor obtenido.

\footnotetext{
12 Júdez, Lucinio (1989): Técnicas de análisis de datos multidimensionales: bases teóricas y aplicaciones en la agricultura, Madrid, MAPA.

${ }^{13}$ Para la aplicación de esta metodología para el desarrollo de indicadores de sostenibilidad véase: Castro, J. Marcos (2002): Indicadores de desarrollo sostenible urbano. Una aplicación para Andalucía, Universidad de Málaga, España, Tesis Doctoral; Soler Rovira, José y Soler Rovira, Pedro A. (2008): “Assessment of aggregated indicators of sustainability using PCA: the case of apple trade in Spain", Zurich, Proceedings of the 6th International Conference on LCA in the Agri-Food Sector.
} 
Tabla 1. Indicadores incluidos en el análisis dentro del marco sistémico de orientadores de Bossell en los tres subsistemas

\begin{tabular}{|c|c|c|c|}
\hline Orientador & Sentido & Indicador & Fuente \\
\hline \multicolumn{4}{|c|}{ Subsistema natural } \\
\hline Existencia & $\begin{array}{lll}\begin{array}{l}\text { Impacto } \\
\text { agricultura }\end{array} & \text { de la }\end{array}$ & $\begin{array}{l}\text { Huella hídrica per cápita de la producción de } \\
\text { alimentos }\end{array}$ & $\begin{array}{l}\text { Waterfootprint } \\
\text { network, FAO }\end{array}$ \\
\hline Efectividad & $\begin{array}{l}\text { Eco-eficiencia de la } \\
\text { agricultura }\end{array}$ & $\begin{array}{l}\text { Huella hídrica de la producción de alimentos } \\
\text { por dólar de valor neto }\end{array}$ & $\begin{array}{l}\text { Waterfootprint } \\
\text { network, FAO }\end{array}$ \\
\hline $\begin{array}{l}\text { Libertad de } \\
\text { acción }\end{array}$ & $\begin{array}{ll}\text { Dependencia } & \mathrm{de} \\
\text { recursos externos } & \end{array}$ & $\begin{array}{l}\text { Ratio entre la huella hídrica del consumo } \\
\text { externa y la interna }\end{array}$ & $\begin{array}{l}\text { Waterfootprint } \\
\text { network }\end{array}$ \\
\hline Seguridad & Recursos propios & $\begin{array}{l}\text { Ratio de la huella hídrica de la producción de } \\
\text { alimentos y las precipitaciones totales de } \\
\text { lluvia }\end{array}$ & $\begin{array}{l}\text { Waterfootprint } \\
\text { network, FAO }\end{array}$ \\
\hline Adaptabilidad & Huella global & $\begin{array}{l}\text { Huella ecológica de la producción de } \\
\text { alimentos }\end{array}$ & $\begin{array}{l}\text { Global footprint } \\
\text { network }\end{array}$ \\
\hline Coexistencia & $\begin{array}{l}\text { Estructura } \quad \text { de } \\
\text { producción }\end{array}$ & Superficie cosechada per cápita & FAO \\
\hline $\begin{array}{l}\text { Necesidades } \\
\text { psicológicas }\end{array}$ & $\begin{array}{l}\text { Limitaciones } \\
\text { ecológicas }\end{array}$ & $\begin{array}{l}\text { Huella ecológica versus biocapacidad } \\
\text { nacional }\end{array}$ & $\begin{array}{l}\text { Global footprint } \\
\text { network }\end{array}$ \\
\hline \multicolumn{4}{|c|}{ Subsistema humano } \\
\hline Existencia & $\begin{array}{lr}\text { Suministro de } & \text { los } \\
\text { requerimientos } & \text { de } \\
\text { ingesta de energía } & \end{array}$ & $\begin{array}{l}\text { Suministro de energía per cápita versus } \\
\text { requerimientos mínimos de ingesta de energía }\end{array}$ & FAO \\
\hline Efectividad & $\begin{array}{l}\text { Eficiencia social de la } \\
\text { actividad agraria }\end{array}$ & $\begin{array}{l}\text { Producción agrícola versus población } \\
\text { empleada en la agricultura }\end{array}$ & FAO \\
\hline $\begin{array}{l}\text { Libertad de } \\
\text { acción }\end{array}$ & $\begin{array}{l}\text { Materias primas en la } \\
\text { dieta }\end{array}$ & $\begin{array}{l}\text { Porcentaje de cereales, raíces y tubérculos en } \\
\text { el suministro de energía en la dieta }\end{array}$ & FAO \\
\hline Seguridad & $\begin{array}{l}\text { Alimentación } \\
\text { completa }\end{array}$ & Consumo total de proteína en la dieta & FAO \\
\hline Adaptabilidad & $\begin{array}{l}\text { Necesidades } \\
\text { alimenticias }\end{array}$ & $\begin{array}{l}\text { Requerimientos mínimos de ingesta de } \\
\text { energía en la dieta }\end{array}$ & FAO \\
\hline Coexistencia & Falta de alimentación & $\begin{array}{l}\text { Déficit de alimentos en la población } \\
\text { desnutrida }\end{array}$ & FAO \\
\hline $\begin{array}{l}\text { Necesidades } \\
\text { psicológicas }\end{array}$ & $\begin{array}{l}\text { Estado personal } \\
\text { percibido }\end{array}$ & Bienestar & $\begin{array}{l}\text { NEF Happy Planet } \\
\text { Index }\end{array}$ \\
\hline \multicolumn{4}{|c|}{ Subsistema productivo } \\
\hline Existencia & Productividad agrícola & Producción de cereales per cápita & FAO \\
\hline Efectividad & Eficiencia productiva & Inversa del rendimiento medio de cereales & FAO \\
\hline $\begin{array}{l}\text { Libertad de } \\
\text { acción }\end{array}$ & $\begin{array}{ll}\text { Dependencia } & \mathrm{de} \\
\text { recursos externos } & \end{array}$ & Importaciones netas de cereales per cápita & FAO \\
\hline
\end{tabular}




\begin{tabular}{|l|lr|l|c|}
\hline Seguridad & $\begin{array}{l}\text { Estabilidad } \\
\text { variabilidad }\end{array}$ & $\mathrm{y}$ & $\begin{array}{l}\text { Estabilidad del rendimiento de los cereales } \\
\text { (coeficiente de variación) }\end{array}$ & FAO \\
\hline Adaptabilidad & $\begin{array}{l}\text { Tecnología } \\
\text { innovación }\end{array}$ & e & $\begin{array}{l}\text { Pendiente de la recta de regresión del } \\
\text { rendimiento de cereales con el tiempo. }\end{array}$ & FAO \\
\hline Coexistencia & $\begin{array}{l}\text { Brecha } \\
\text { productividad }\end{array}$ & de & $\begin{array}{l}\text { Brecha del rendimiento de cereales (\% del } \\
\text { rendimiento máximo versus el medio) }\end{array}$ & FAO \\
\hline $\begin{array}{l}\text { Necesidades } \\
\text { psicológicas }\end{array}$ & $\begin{array}{l}\text { Trabajo en el } \\
\text { agricultura }\end{array}$ & Trabajadores agrícolas por hectárea & FAO \\
\hline
\end{tabular}

Fuentes empleadas $^{14}$

Tabla 2. Relación de países incluidos en el estudio

\begin{tabular}{|c|c|c|c|c|c|c|c|c|}
\hline País & Magreb & Máshreq & Oriente & Europa & U.E. & Mediterráneo & Sahel & OPEC \\
\hline & & & & & & & & \\
\hline Albania & & & & $\mathrm{X}$ & candidato & $X$ & & \\
\hline Alemania & & & & $\mathrm{X}$ & $\mathrm{X}$ & & & \\
\hline Angola & & & & & & & & $X$ \\
\hline Arabia Saudí & & $X$ & $\mathrm{X}$ & & & & & $\mathrm{X}$ \\
\hline Argelia & $X$ & & & & & $X$ & $X$ & $\mathrm{X}$ \\
\hline Austria & & & & $X$ & $X$ & & & \\
\hline Bélgica & & & & $X$ & $X$ & & & \\
\hline Bosnia & & & & $X$ & candidato & $X$ & & \\
\hline Bulgaria & & & & $X$ & $\mathrm{X}$ & & & \\
\hline Chad & & & & & & & $X$ & \\
\hline Chipre & & & $\mathrm{X}$ & $X$ & $\mathrm{X}$ & $X$ & & \\
\hline Croacia & & & & $X$ & adhesión & $X$ & & \\
\hline Dinamarca & & & & $X$ & $\mathrm{X}$ & & & \\
\hline Ecuador & & & & & & & & $X$ \\
\hline Eginto & & $\mathrm{X}$ & $X$ & & & $X$ & & \\
\hline Emiratos Árabes & & $\mathrm{X}$ & $X$ & & & & & $\mathrm{X}$ \\
\hline Eritrea & & & & & & & $\mathrm{X}$ & \\
\hline Eslovaquia & & & & X & $X$ & & & \\
\hline Eslovenia & & & & $\mathrm{X}$ & $X$ & $X$ & & \\
\hline España & & & & $X$ & $X$ & $X$ & & \\
\hline Estonia & & & & $X$ & $X$ & & & \\
\hline Finlandia & & & & $X$ & $X$ & & & \\
\hline Francia & & & & $\mathrm{X}$ & $X$ & $X$ & & \\
\hline Grecia & & & & $X$ & $X$ & $X$ & & \\
\hline Holanda & & & & $X$ & $X$ & & & \\
\hline Hungría & & & & $\mathrm{X}$ & $\mathrm{X}$ & & & \\
\hline Irán & & & $X$ & & & & & $\mathrm{X}$ \\
\hline Irlanda & & & & $X$ & $\mathrm{X}$ & & & \\
\hline Israel & & & $X$ & & & $X$ & & \\
\hline Italia & & & & $X$ & $X$ & $X$ & & \\
\hline Jordania & & $X$ & $\mathrm{X}$ & & & & & \\
\hline Kuwait & & $X$ & $\mathrm{X}$ & & & & & $\mathrm{X}$ \\
\hline Letonia & & & & $X$ & $X$ & & & \\
\hline Líbano & & $X$ & $\mathrm{X}$ & & & $X$ & & \\
\hline Libia & $X$ & & & & & $X$ & & $\mathrm{X}$ \\
\hline Lituania & & & & $X$ & $X$ & & & \\
\hline Luxemburgo & & & & $\mathrm{X}$ & $\mathrm{X}$ & & & \\
\hline Macedonia & & & & $\mathrm{X}$ & candidato & & & \\
\hline
\end{tabular}

\footnotetext{
${ }^{14}$ Las fuentes citadas en la tabla que se han empleado para la elaboración de los indicadores son: FAO (2012): El Estado de la Inseguridad Alimentaria en el Mundo 2012, en http://www.fao.org/publications/sofi/food-securityindicators/es/; FAO (2012): FAOSTAT, en http://faostat.fao.org/default.aspx?lang=es; Footprint of Nations, en: http://www.footprintnetwork.org/en/index.php/GFN/page/footprint_for_nations/; Happy Planet Index (2012): The Data, en http://www.happyplanetindex.org/data/; Water footprint of nations (2012), en http://www.waterfootprint.org.
} 


\begin{tabular}{|c|c|c|c|c|c|c|c|c|}
\hline Malí & & & & & & & $\mathrm{X}$ & \\
\hline Malta & & & & $\mathrm{X}$ & $\mathrm{X}$ & $\mathrm{X}$ & & \\
\hline Marruecos & $X$ & & & & & $\mathrm{X}$ & & \\
\hline Mauritania & $\mathrm{X}$ & & & & & & $\mathrm{X}$ & \\
\hline México & & & & & & & & \\
\hline Níger & & & & & & & $\mathrm{X}$ & \\
\hline Nigeria & & & & & & & & $\mathrm{X}$ \\
\hline Noruega & & & & $\mathrm{X}$ & & & & \\
\hline Polonia & & & & $\mathrm{X}$ & $\mathrm{X}$ & & & \\
\hline Portugal & & & & $\mathrm{X}$ & $\mathrm{X}$ & & & \\
\hline República Checa & & & & $X$ & $X$ & & & \\
\hline Rumanía & & & & $\mathrm{X}$ & $X$ & & & \\
\hline Senegal & & & & & & & $X$ & \\
\hline Serbia & & & & $X$ & candidato & $\mathrm{X}$ & & \\
\hline Sudáfrica & & & & & & & & \\
\hline Sudán & & $\mathrm{X}$ & & & & & $\mathrm{X}$ & \\
\hline Suecia & & & & $X$ & $X$ & & & \\
\hline Siria & & $\mathrm{X}$ & $\mathrm{X}$ & & & $\mathrm{X}$ & & \\
\hline Túnez & $\mathrm{X}$ & & & & & $\mathrm{X}$ & & \\
\hline Turquía & & & $\mathrm{X}$ & & candidato & $\mathrm{X}$ & & \\
\hline Reino Unido & & & & $X$ & $\mathrm{X}$ & & & \\
\hline Yemen & & $X$ & $X$ & & & & & \\
\hline
\end{tabular}

\section{Resultados v discusión}

Los indicadores seleccionados explicaron satisfactoriamente las diferencias en seguridad alimentaria sostenible entre los países estudiados, así como para cada dimensión de la sostenibilidad.

En la tabla 3 se muestran los resultados del análisis de componentes principales realizado, en el que se retuvieron seis componentes que explican un $79,8 \%$ de la varianza total de los datos. El primer componente (CP1) está altamente correlacionado con seis indicadores relacionados con el subsistema humano y la seguridad alimentaria y tres con la efectividad productiva y ecológica. El segundo componente $(\mathrm{CP} 2)$ está relacionado con indicadores de existencia y libertad de acción de los subsistemas natural y productivo. El tercer componente lo está con indicadores de seguridad y coexistencia del subsistema productivo; el cuarto con la efectividad del subsistema humano y la adaptabilidad natural, el quinto con la estructura de la producción de alimentos (superficie per cápita) y el sexto con los indicadores de recursos hídricos propios y la introducción de tecnología en la agricultura.

Estos indicadores explican satisfactoriamente las diferencias en seguridad alimentaria sostenible entre los países estudiados, y las coordenadas de cada país con cada componente principal contribuirán al valor del indicador agregado, ponderado con el autovalor de cada componente. El valor del indicador resultante y el orden de prelación establecido entre los 60 países figuran en la tabla 4. Treinta y cuatro países muestran un valor positivo del indicador de seguridad alimentaria sostenible, por lo que puede considerarse que en esos países, y teniendo en cuenta las premisas y datos de partida de este trabajo, sí existe una seguridad alimentaria sostenible relativa, mientras que en el resto no se asegura esa sostenibilidad ya que presentan un valor del indicador negativo. Túnez, Argelia, y Marruecos muestran valores negativos del indicador, siendo dichos valores más altos, en valor absoluto, en este orden, por lo que presentan una insostenibilidad relativa respecto al resto de países incluidos en el estudio, como por ejemplo España que muestra un valor positivo. Los países que se encuentran en las primeras posiciones son países de la Unión Europea, y en las últimas se sitúan países del Sahel, Oriente próximo y Angola que pertenece a la OPEC. España figura en 
el puesto 22 de los 60 países incluidos en este estudio, mientras que Túnez ocupa el puesto 39, Argelia el 46 y Marruecos el 50.

Tabla 3. Resultados del análisis de componentes principales

\begin{tabular}{|c|c|c|c|c|c|c|}
\hline $\begin{array}{l}\text { Componentes } \\
\text { principales }\end{array}$ & CP1 & CP2 & CP3 & CP4 & CP5 & CP6 \\
\hline Autovalor & 7,72 & 3,54 & 1,81 & 1,57 & 1,07 & 1,04 \\
\hline $\begin{array}{l}\text { Varianza } \\
\text { explicada }(\%)\end{array}$ & 36,7 & 16,9 & 8,6 & 7,5 & 5,1 & 5,0 \\
\hline \multirow[t]{6}{*}{$\begin{array}{l}\text { Indicadores } \\
\text { correlacionados } \\
\text { (coeficiente de } \\
\text { correlación con } \\
\text { cada CP) }\end{array}$} & $\begin{array}{c}\text { Déficit de } \\
\text { alimentos en la } \\
\text { población } \\
\text { desnutrida } \\
(0,92)\end{array}$ & $\begin{array}{c}\text { Huella hídrica } \\
\text { per cápita de } \\
\text { la producción } \\
\text { de alimentos } \\
\quad(-0,87)\end{array}$ & $\begin{array}{l}\text { Estabilidad } \\
\text { del } \\
\text { rendimiento } \\
\text { de los } \\
\text { cereales } \\
(0,94)\end{array}$ & $\begin{array}{c}\text { Huella } \\
\text { ecológica } \\
\text { de la } \\
\text { producción } \\
\text { de } \\
\text { alimentos } \\
(0,83)\end{array}$ & $\begin{array}{c}\text { Superficie } \\
\text { cosechada } \\
\text { per cápita } \\
(-0,72)\end{array}$ & $\begin{array}{c}\text { Ratio de la } \\
\text { huella hídrica } \\
\text { de la } \\
\text { producción de } \\
\text { alimentos y las } \\
\text { precipitaciones } \\
\text { totales de } \\
\text { lluvia } \\
(0,85)\end{array}$ \\
\hline & $\begin{array}{l}\text { Inversa del } \\
\text { rendimiento } \\
\text { medio de } \\
\text { cereales } \\
(0,84)\end{array}$ & $\begin{array}{l}\text { Importaciones } \\
\text { netas de } \\
\text { cereales per } \\
\text { cápita } \\
(0,86)\end{array}$ & $\begin{array}{c}\text { Brecha del } \\
\text { rendimiento } \\
\text { de cereales } \\
(0,92)\end{array}$ & $\begin{array}{c}\text { Producción } \\
\text { agrícola } \\
\text { versus } \\
\text { población } \\
\text { empleada } \\
\text { en la } \\
\text { agricultura } \\
(-0,55)\end{array}$ & & $\begin{array}{l}\text { Pendiente de } \\
\text { la recta de } \\
\text { regresión del } \\
\text { rendimiento de } \\
\text { cereales con el } \\
\text { tiempo } \\
(0,59)\end{array}$ \\
\hline & $\begin{array}{l}\text { Requerimientos } \\
\text { mínimos de } \\
\text { ingesta de } \\
\text { energía en la } \\
\text { dieta } \\
(0,83)\end{array}$ & $\begin{array}{c}\text { Ratio entre la } \\
\text { huella hídrica } \\
\text { del consumo } \\
\text { externa y la } \\
\text { interna } \\
(0,75)\end{array}$ & & & & \\
\hline & $\begin{array}{l}\text { Suministro de } \\
\text { energía per } \\
\text { cápita versus } \\
\text { requerimientos } \\
\text { mínimos de } \\
\text { ingesta de } \\
\text { energía } \\
(0,82)\end{array}$ & $\begin{array}{c}\text { Producción } \\
\text { de cereales } \\
\text { per cápita } \\
(0,74)\end{array}$ & & & & \\
\hline & $\begin{array}{c}\text { Consumo total } \\
\text { de proteína en } \\
\text { la dieta } \\
(0,80)\end{array}$ & $\begin{array}{c}\text { Huella } \\
\text { ecológica } \\
\text { versus } \\
\text { biocapacidad } \\
\text { nacional } \\
(0,71)\end{array}$ & & & & \\
\hline & $\begin{array}{l}\text { Porcentaje de } \\
\text { cereales, raíces } \\
\text { y tubérculos en }\end{array}$ & & & & & \\
\hline
\end{tabular}




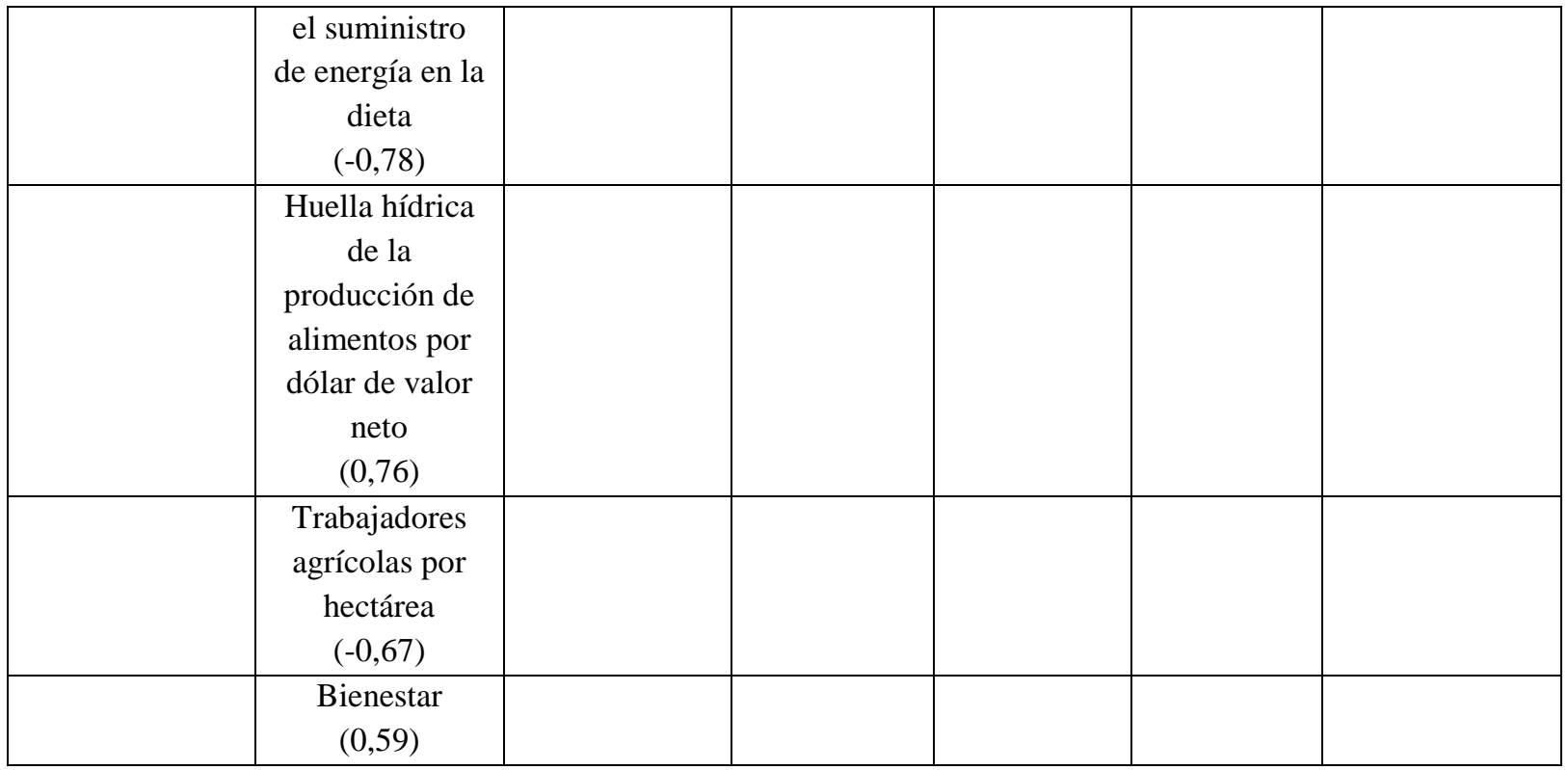

Tabla 4. Valores para cada país de los indicadores agregados de seguridad alimentaria sostenible, sostenibilidad ambiental, social y productiva (en cursiva los países con valores positivos de cada indicador agregado; en negrita se han marcado a Argelia, Marruecos, Túnez y España)

\begin{tabular}{|c|c|c|c|c|c|c|c|}
\hline País & $\mathrm{ACP}_{\text {seauridadalimentariasostenible. }}$ & País & $\mathrm{ACP}_{\text {sost ambiental }}$ & País & $\mathrm{ACP}_{\text {sostsocial }}$ & País & $\mathrm{ACP}_{\text {sost nroductiva }}$ \\
\hline Dinamarca & 3.47 & Níaer & 3.26 & Luxemburao & 7.35 & Francia & 2.72 \\
\hline Francia & 3.28 & Chad & 2.36 & Dinamarca & 7.24 & Dinamarca & 2.64 \\
\hline Hunaría & 2.71 & Ecuador & 1.75 & Bélqica & 6.66 & Hunaría & 2.23 \\
\hline Austria & 2.14 & Malí & 1.73 & Holanda & 6.05 & Finlandia & 1.59 \\
\hline Alemania & 2.12 & Seneqal & 1.65 & Austria & 6.03 & Alemania & 1.53 \\
\hline Luxemburqo & 2.06 & Eritrea & 1.60 & Francia & 6.03 & Rep. Checa & 1.38 \\
\hline Finlandia & 2.06 & Sudán & 1.55 & Israel & 5.76 & Letonia & 1.38 \\
\hline Rep.Checa & 1.87 & Anqola & 1.51 & Alemania & 5.20 & Reino Unido & 1.38 \\
\hline Lituania & 1.80 & Rumania & 1.40 & Irlanda & 5.18 & Suecia & 1.37 \\
\hline Suecia & 1.75 & Serbia v M. & 1.32 & Italia & 4.91 & Serbia v M. & 1.24 \\
\hline Irlanda & 1.73 & Hunaría & 1.28 & Eslovenia & 4.78 & Italia & 1.23 \\
\hline Grecia & 1.70 & Lituania & 1.25 & Reino Unido & 4.74 & Luxemburqo & 1.17 \\
\hline Reino Unido & 1.66 & Sudáfrica & 1.21 & Norueqa & 4.65 & Irlanda & 1.11 \\
\hline Italia & 1.63 & Nigeria & 1.13 & Grecia & 4.50 & Austria & 1.07 \\
\hline Bélquica & 1.49 & Bulqaria & 1.02 & Malta & 4.41 & Polonia & 1.07 \\
\hline Polonia & 1.41 & Letonia & 0.90 & Suecia & 4.22 & Grecia & 1.02 \\
\hline Eslovenia & 1.27 & Siria & 0.90 & España & 4.07 & Croacia & 0.99 \\
\hline Letonia & 1.24 & Turquía & 0.86 & Finlandia & 4.01 & Lituania & 0.98 \\
\hline Rumania & 1.21 & Marruecos & 0.84 & Portuqal & 3.07 & Estonia & 0.94 \\
\hline Croacia & 1.17 & Irán & 0.81 & Chipre & 2.94 & Turquía & 0.91 \\
\hline Norueqa & 1.12 & Túnez & 0.77 & Rep. Checa & 2.68 & México & 0.83 \\
\hline España & 1.07 & Eslovaquia & 0.73 & Lituania & 2.29 & Eslovenia & 0.79 \\
\hline Serbia v $M$. & 1.07 & Croacia & 0.72 & Em. Árabes Un. & 2.23 & Bulqaria & 0.76 \\
\hline Estonia & 1.03 & Estonia & 0.50 & Rumania & 2.09 & Ecuador & 0.66 \\
\hline Holanda & 0.79 & Polonia & 0.48 & Polonia & 2.07 & Bélqica & 0.57 \\
\hline Malta & 0.76 & México & 0.46 & Hunaría & 1.94 & Eslovaquia & 0.51 \\
\hline Turquía & 0.69 & Mauritania & 0.32 & Estonia & 0.48 & Macedonia & 0.50 \\
\hline Portuqal & 0.68 & Suecia & 0.25 & Kuwait & 0.44 & Bosnia v $\mathrm{H}$. & 0.50 \\
\hline Eslovaquia & 0.66 & Bosnia v $\mathrm{H}$. & 0.20 & Turquía & 0.43 & Rumania & 0.37 \\
\hline Bulqaria & 0.65 & República & 0.17 & Croacia & 0.25 & España & 0.32 \\
\hline Bosnia v $H$. & 0.43 & Albania & 0.16 & México & -0.03 & Niqeria & 0.30 \\
\hline Macedonia & 0.33 & Yemen & 0.14 & Eslovaquia & -0.04 & Norueqa & 0.30 \\
\hline México & 0.27 & Finlandia & 0.14 & Líbano & -0.28 & Portuqal & 0.29 \\
\hline Israel & 0.04 & Reino Unido & 0.10 & Letonia & -0.36 & Malta & 0.26 \\
\hline Albania & -0.21 & Arqelia & 0.06 & Bosnia y $\mathrm{H}$. & -0.46 & Irán & 0.19 \\
\hline Sudáfrica & -0.32 & Macedonia & -0.12 & Albania & -0.50 & Líbano & 0.17 \\
\hline Líbano & -0.34 & Francia & -0.19 & Túnez & -0.53 & Eqipto & 0.01 \\
\hline Ecuador & -0.37 & Eslovenia & -0.26 & Macedonia & -1.05 & Holanda & -0.14 \\
\hline Túnez & -0.55 & España & -0.29 & Arabia Saudí & -1.43 & Arabia Saudí & -0.20 \\
\hline Irán & -0.62 & Grecia & -0.48 & Serbia v M. & -1.44 & Em.ÁrabesUn. & \\
\hline Em. Árabes Un. & -0.68 & Libia & -0.57 & Libia & -1.44 & Sudáfrica & -0.22 \\
\hline
\end{tabular}




\begin{tabular}{|c|c|c|c|c|c|c|c|}
\hline Kuwait & -0.95 & Austria & -0.64 & Jordania & -2.10 & Chad & -0.37 \\
\hline Arabia Saudí & -0.99 & Portugal & -0.73 & Sudáfrica & -2.30 & Kuwait & -0.55 \\
\hline Eqipto & -1.05 & Eqipto & -0.74 & Bulqaria & -2.46 & Israel & -0.5 \\
\hline Chipre & -1.10 & Irlanda & -0.83 & Arqelia & -2.63 & Túnez & -0.7 \\
\hline Arqelia & -1.28 & Arabia Saudí & -0.85 & Irán & -3.00 & Libia & -0.7 \\
\hline Libia & -1.48 & Italia & -1.01 & Siria & -3.02 & Albania & -0.9 \\
\hline Niqeria & -1.48 & Alemania & -1.04 & Marruecos & -3.19 & Malí & -1.0 \\
\hline Siria & -1.66 & Chipre & -1.21 & Mauritania & -3.69 & Níger & -1.0 \\
\hline Marruecos & -1.92 & Norueqa & -1.31 & Eqipto & -3.87 & Arqelia & -1.1 \\
\hline Malí & -1.92 & Líbano & -1.51 & Ecuador & -4.31 & Siria & -1.4 \\
\hline Mauritania & -2.32 & Dinamarca & -1.77 & Niqeria & -5.87 & Seneaal & -1.6 \\
\hline Jordania & -2.59 & Jordania & -1.85 & Sudán & -6.36 & Sudán & -1.7 \\
\hline Sudán & -2.74 & Malta & -2.08 & Malí & -7.19 & Yemen & -2.1 \\
\hline Seneaal & -2.76 & Luxemburqo & -2.18 & Níqer & -7.47 & Mauritania & -2.3 \\
\hline Chad & -2.78 & Em. Arabes Un. & -2.37 & Seneqal & -7.97 & Angola & -2. \\
\hline Níger & -3.15 & Holanda & -2.59 & Yemen & -9.57 & Marruecos & -2.7 \\
\hline Angola & -3.64 & Bélqiica & -2.80 & Chad & -10.17 & Chipre & -3.7 \\
\hline Yemen & -3.87 & Israel & -2.95 & Anqola & -10.78 & Jordania & -4 \\
\hline Eritrea & -6.60 & Kuwait & -3.18 & Eritrea & -13.15 & Eritrea & \\
\hline
\end{tabular}

Sin embargo, en lo que se refiere a la sostenibilidad ambiental, los países del Magreb Central muestran valores positivos (tabla 4), y por lo tanto presentan una sostenibilidad ambiental o ecológica relativa, mientras que España presenta un valor negativo, lo que evidencia una insostenibilidad ambiental relativa. En la tabla 5 figuran los valores de las variables incluidas en los tres subsistemas, a los que se les ha restado la media y se les ha dividido por la desviación típica (valores estandarizados). Cuando éstos toman valores positivos o mayores de uno quiere decir que esas variables contribuyen positivamente a esa dimensión de la sostenibilidad y, en consecuencia, al indicador correspondiente. Por el contrario, cuando los valores de la tabla 5 son negativos, esas variables contribuyen negativamente a la dimensión e indicador asociado considerados. De este modo, se pueden evaluar las fortalezas y debilidades de cada país en cada dimensión de la sostenibilidad.

En la dimensión ambiental o subsistema natural (tabla 5), Túnez y Marruecos presentan valores positivos en el ratio huella hídrica externa e interna, huella ecológica en la producción de alimentos, huella versus biocapacidad y superficie cosechada per cápita. Estas variables son las que contribuyen a que estos países tengan un valor positivo del indicador agregado de sostenibilidad ambiental (tabla 4) y que se puedan considerar ambientalmente sostenibles de modo relativo (con las premisas tenidas en cuenta en este estudio). Las fortalezas de Argelia pueden concretarse en menores huellas hídrica y ecológica en la producción de alimentos. Estas variables actuarían como debilidades en el caso de España, por lo que el indicador agregado de sostenibilidad ambiental resulta ser negativo y, por lo tanto, se considere ambientalmente insostenible de modo relativo.

En cuanto al subsistema humano o dimensión social o de seguridad alimentaria, los países del Magreb Central muestran valores negativos del indicador agregado de sostenibilidad social (tabla 4), situándose en la zona de insostenibilidad relativa. Los peor situados son Marruecos (puesto 49) y Argelia (puesto 46), ya que presentan valores negativos en las variables bienestar, requerimientos de energía y proteína y déficit de alimentos en la población desnutrida (tabla 5). Por otro lado, tienen una baja productividad de la mano de obra agrícola y los cereales representan un porcentaje importante del aporte total de energía. Túnez se sitúa en el puesto 38, con debilidad también en las variables bienestar y eficiencia.

En la dimensión productiva o subsistema productivo aparece una distribución similar a la del subsistema humano pero con posiciones más bajas en el ranking relativo de los países en función del valor del indicador agregado de sostenibilidad económica (tabla 4). Las 
variables que contribuyen a esta insostenibilidad productiva relativa son la producción de cereales per cápita, el rendimiento medio, su estabilidad y la brecha existente respecto al máximo potencial y las necesidades asociadas de importarlos del exterior. En este sentido, se muestran como debilidades de los países del Magreb Central la baja productividad y estabilidad de la producción de cereales y la dependencia del exterior para su suministro, factores ya apuntados para el Magreb y, en concreto, para Argelia ${ }^{15}$.

Tabla 5. Valores de las variables de los tres subsistemas de los países del Magreb Central y España (valores estandarizados: valor menos la media y dividido entre la desviación típica)

\begin{tabular}{|c|c|c|c|c|}
\hline Variable & Argelia & Marruecos & Túnez & España \\
\hline \multicolumn{5}{|l|}{ Subsistema natural o dimensión ambiental } \\
\hline Huella hídrica per cápita de la producción de alimentos & 0.77 & -0.04 & -1.04 & -0.98 \\
\hline $\begin{array}{l}\text { Huella hídrica de la producción de alimentos por dólar de } \\
\text { valor neto }\end{array}$ & 0.03 & -0.26 & -0.42 & 0.51 \\
\hline $\begin{array}{l}\text { Ratio entre la huella hídrica del consumo externa y la } \\
\text { interna }\end{array}$ & -0.25 & 0.62 & 0.49 & 0.08 \\
\hline $\begin{array}{l}\text { Ratio de la huella hídrica de la producción de alimentos y } \\
\text { las precipitaciones totales de lluvia }\end{array}$ & 0.45 & -0.23 & -1.86 & -0.30 \\
\hline Huella ecológica de la producción de alimentos & 0.93 & 0.90 & 0.75 & -0.95 \\
\hline Superficie cosechada per cápita & -0.38 & 0.19 & 0.63 & 0.41 \\
\hline Huella ecológica versus biocapacidad nacional & 0.12 & 0.33 & 0.35 & -0.09 \\
\hline \multicolumn{5}{|l|}{ Subsistema humano o dimensión social } \\
\hline $\begin{array}{l}\text { Suministro de energía per cápita versus requerimientos } \\
\text { mínimos de ingesta de energía }\end{array}$ & 0.30 & 0.64 & 0.72 & 0.22 \\
\hline $\begin{array}{l}\text { Producción agrícola versus población empleada en la } \\
\text { agricultura }\end{array}$ & -0.72 & -0.71 & -0.64 & 0.55 \\
\hline $\begin{array}{l}\text { Porcentaje de cereales, raíces y tubérculos en el } \\
\text { suministro de energía en la dieta }\end{array}$ & 1.21 & 1.50 & 0.72 & -1.24 \\
\hline Consumo total de proteína en la dieta & -0.23 & -0.10 & 0.10 & 0.92 \\
\hline Requerimientos mínimos de ingesta de energía en la dieta & -0.64 & -0.42 & -0.19 & 0.69 \\
\hline Déficit de alimentos en la población desnutrida & -0.38 & -0.73 & 0.67 & 0.67 \\
\hline Bienestar & -0.28 & -0.99 & -0.74 & 0.50 \\
\hline \multicolumn{5}{|l|}{ Subsistema productivo o dimensión económica } \\
\hline Producción de cereales per cápita & -0.76 & -0.42 & -0.59 & 0.29 \\
\hline Inversa del rendimiento medio de cereales & -0.44 & -0.53 & -0.27 & 0.42 \\
\hline Importaciones netas de cereales per cápita & -0.69 & -0.37 & -0.89 & -0.73 \\
\hline $\begin{array}{l}\text { Estabilidad del rendimiento de los cereales (coeficiente de } \\
\text { variación) }\end{array}$ & -0.51 & -2.94 & -0.21 & 0.06 \\
\hline $\begin{array}{l}\text { Pendiente de la recta de regresión del rendimiento de } \\
\text { cereales con el tiempo. }\end{array}$ & 0.23 & 1.23 & 0.94 & -0.85 \\
\hline
\end{tabular}

\footnotetext{
15 "Suministro de Alimentos, Factores Agronómicos...", op. cit.; "Suministro de Alimentos, Insumos...", op. cit.
} 
Brecha del rendimiento de cereales (\% del rendimiento máximo versus el medio)

Trabajadores agrícolas por hectárea

$-0.24$

0.33
$-0.32$

$-0.33$
$-0.24$

$-0.68$

\section{Conclusiones}

Los países del Magreb Central (Argelia, Marruecos y Túnez) muestran valores negativos del indicador agregado de seguridad alimentaria sostenible estimado utilizando la técnica de análisis multivariante de análisis de componentes principales, por lo que presentan una inseguridad e insostenibilidad relativas respecto al resto de los países incluidos en este estudio (60 en total). Por el contrario España u otros países de la UE muestran un valor positivo, y por lo tanto una seguridad alimentaria sostenible relativa. Así, los países que se encuentran en las primeras posiciones con los valores del indicador más altos son países de la Unión Europea, y, en las últimas con valores negativos, países del Sahel, Oriente próximo y Magreb.

Con relación a las tres dimensiones de la sostenibilidad, los países del Magreb Central presentan valores positivos en el indicador agregado de sostenibilidad ambiental, aunque son negativos en los indicadores de sostenibilidad social y productiva. Las variables que explican esta fortaleza en la sostenibilidad ambiental son las menores huellas hídrica y ecológica en la producción de alimentos que la que muestran otros países incluidos en este estudio. Por otra parte, estos tres países presentan valores que contribuyen negativamente a la sostenibilidad social y seguridad alimentaria en las variables de requerimientos de alimentos, energía y proteína y también en el bienestar percibido por sus poblaciones. Por otro lado, los cereales representan un porcentaje importante del aporte total de energía en la dieta y, paralelamente, los rendimientos son bajos y variables, dependiendo el suministro interno de las importaciones del exterior, lo que les hace ser relativamente insostenibles desde el punto de vista productivo.

Por lo tanto, los países del Magreb Central no muestran una seguridad alimentaria sostenible de acuerdo a las variables estudiadas y dentro del conjunto de países incluidos en este trabajo. Aunque la producción de alimentos es relativamente sostenible desde el punto de vista ambiental, no lo es en las dimensiones productiva y humana o social, ya que los cereales se presentan como el mayor aporte energético en la dieta, si bien, por un lado, no cubren los requerimientos óptimos y, por otro, la productividad de su cultivo en el Magreb es deficiente, debiéndose cubrir con importaciones externas.Este análisis sigue el trabajo emprendido por Soler Rovira et al. ${ }^{16}$, y supone otro paso para poder avanzar en desarrollo de una producción de alimentos sostenible que asegure la alimentación de la población de os países del Magreb Central.

\footnotetext{
16 Soler-Rovira, José; Arroyo-Sanz, Juan Manuel; González-Torres, Francisco; Rojo-Hernández, Carlos y Marquina Barrio, Antonio: "Aggregated indicators of sustainable agricultural production and food security in the Central Maghreb", en Corson, M.S.; van der Werf, H.M.G. (eds.) (2012): Book of Abstracts of the $8^{\text {th }}$ International Conference on Life Cycle Assessment in the Agri-Food Sector (LCA Food 2012), Saint-Malo, France, 2012.
} 